\title{
NOTES
}

\section{Narrowly Spaced Comb-Like Polymers}

\author{
Tadamichi HIRABAYASHI and Kenji YoKota \\ Department of Materials Science and Engineering, \\ Nagoya Institute of Technology, \\ Gokiso-cho, Showa-ku, Nagoya 466, Japan
}

(Received March 26, 1987)

\begin{abstract}
KEY WORDS Comb-Like Polymer / Narrowly Spaced Comb-Like Polymer / Dialkyl Fumarate / Dialkyl Itaconate / Long Alkyl Group / Crystallization /
\end{abstract}

In our previous papers ${ }^{1-3}$ we observed the side chain crystallization of several widely spaced comb-like polymers in which their teeth (long alkyl side chains) appeared regularly on every 6 , and $11-15$ main chain atoms in spite of their wide spaces. The structure and extent of crystallization were similar to those of the conventional comb-like polymers ${ }^{4}$ in which the teeth appear on every 2 main chain carbon atoms.

In this note we report the side chain crystallization of polydialkyl fumarates and itaconates. We designate these polymers as narrowly spaced comb-like polymers because each of them has two long alkyl side chains on every 2 main chain carbon atoms.

\section{EXPERIMENTAL}

\section{Monomers}

Dialkyl fumarates were obtained in high yield from fumaroyl chloride and fatty alcohol. Recrystallized products from acetone showed the following mp: didodecyl fumarate $45-46^{\circ} \mathrm{C}$, ditetradecyl fumarate $57-58^{\circ} \mathrm{C}$, dihexadecyl fumarate $66-66.5^{\circ} \mathrm{C}$, and dioctadecyl fumarate $70-72^{\circ} \mathrm{C}$.

Dialkyl itaconates were obtained in 50 $80 \%$ yield from itaconic anhydride and fatty alcohol in refluxing benzene with $p$ r toluenesulfonic acid catalyst. Water was removed by using a Dean-Stark trap. Pure dialkyl itaconates were obtained by recrystallizing from benzene. Didodecyl itaconate was purified by rapid vacuum distillation. $\mathrm{mp}$ are: didodecyl itaconate $c a .21^{\circ} \mathrm{C}$, ditetradecyl itaconate $41-42^{\circ} \mathrm{C}$, dihexadecyl itaconate $51-52^{\circ} \mathrm{C}$, and dioctadecyl itaconate $57-$ $58^{\circ} \mathrm{C}$.

Hexadecyl methyl itaconate (or hexadecyl 3methoxycarbonyl-3-butenoate), was obtained as follows. An equimolar mixture of itaconic anhydride and hexadecyl alcohol in benzene was warmed to a clear solution and stood overnight at room temperature. White crystals were collected and recrystallized from acetone. Hexadecyl hydrogen itaconate, obtained in $50 \%$ yield, was reacted in benzene with a small excess of diazomethane. Benzene was removed in vacuum and a low melting product was obtained quantitatively. It was satisfactorily pure and used as such for polymerization. Attempted vacuum distillation caused isomerization.

The structure and purity of each monomer were confirmed by infrared (IR) and ${ }^{1} \mathrm{H}$ nuclear magnetic resonance (NMR) spectra. 


\section{Polymerization}

Polymerization of dialkyl fumarates was carried out as follows. A mixture of a dialkyl fumarate $(2 \mathrm{~g})$ and $2,2^{\prime}$-azobisisobutyronitrile $(50 \mathrm{mg})$ was warmed at $80^{\circ} \mathrm{C}$ for 5 days with occasional shaking. The viscous liquid was dissolved in a small volume of benzene and precipitated into methanol. The collected solid was stirred for $10 \mathrm{~min}$ with hot ethanol $(100 \mathrm{ml})$ and the supernatant was discarded while hot. This extraction procedure was repeated ten times to remove the unreacted monomer completely. Polydialkyl fumarates were obtained in $30-40 \%$ conversion after vacuum drying.

Polymerization of dialkyl itaconates was similar to the above. Dialkyl itaconate $(4 \mathrm{~g})$ was warmed with $2,2^{\prime}$-azobisisobutyronitrile $(20 \mathrm{mg})$ in toluene $(2 \mathrm{ml})$ at $60^{\circ} \mathrm{C}$ for 2 days. No toluene was added for didodecyl monomers. Polydidodecyl itaconate was purified by repeated reprecipitations from benzene into methanol. Polyditetradecyl and dihexadecyl itaconates were purified by repeated reprecipitations from benzene into acetone. Polydioctadecyl itaconate was purified by hot ethanol extraction. Polydialkyl itaconates were obtained in $50-60 \%$ conversion after vacuum drying.

Polymerization of hexadecyl methyl itaconate was carried out in the same manner with didodecyl itaconate and obtained in $56 \%$ conversion.

\section{Measurements}

Gel-permeation chromatography (GPC) for molecular weight determination, differential scanning calorimetry (DSC) for thermal analysis, X-ray diffraction and IR studies for crystalline structure were the same as in previous papers. ${ }^{1-3}$

\section{RESULTS AND DISCUSSION}

The structures of polymers prepared as above were all identified by their IR, ${ }^{1} \mathrm{H}$ and
${ }^{13} \mathrm{C}$ NMR spectra.

Dialkyl fumarates have long been classified as monomers which hardly polymerize and give only low molecular weight polymers owing to their 1,2-disubstituted structure. Recently, however, Otsu and his coworkers ${ }^{5-7}$ found that branched dialkyl fumarates can easily give high molecular weight polymers. The present fumarates are not branched, but

Table I. Molecular weights of comb-like polymer samples

\begin{tabular}{lcc}
\hline Polymer & $\begin{array}{c}\text { Molecular weight } \\
\times 10^{-4}\end{array}$ & $\begin{array}{c}\text { Degree of } \\
\text { polymerization }\end{array}$ \\
\hline Polyfumarates & & \\
Didodecyl & 1.05 & 23 \\
Ditetradecyl & 1.05 & 21 \\
Dihexadecyl & 0.97 & 17 \\
Dioctadecyl & 1.05 & 17 \\
Polyitaconates & & \\
Didodecyl & 3.90 & 84 \\
Ditetradecyl & 2.10 & 40 \\
Dihexadecyl & 1.60 & 28 \\
Dioctadecyl & 0.97 & 15 \\
Polyitaconate & & 141 \\
Hexadecyl methyl & 5.20 & \\
\hline
\end{tabular}

a From GPC peak top.

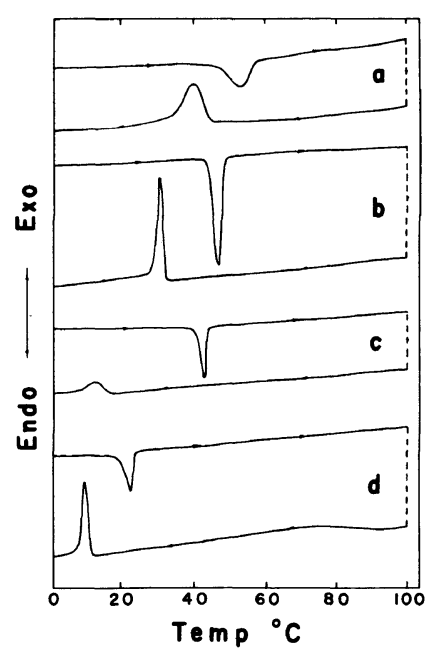

Figure 1. DSC traces for (a) polydihexadecyl fumarate, (b) polydihexadecyl itaconate, (c) polyhexadecyl methyl itaconate, and (d) polyhexadecyl methacrylate. 
give fairly high molecular weight polymers as shown in Table I. Table I also shows that the molecular weights of polydialkyl itaconates are not so much high, because of partial chain transfer to monomers. ${ }^{8,9}$ According to our previous results on poly-2-hexadecyltrimethylene alkanedioates, ${ }^{3}$ the molecular weights of polymer samples in Table I seems high enough to discuss the side chain crystallization.

Figure 1 shows heating and cooling DSC traces for four types of hexadecyl polymers of fumarate, itaconate, 3-methoxycarbonyl-3butenoate, and methacrylate. The former two are narrowly spaced comb-like polymers and the latter two are conventional comb-like polymers. Early studies ${ }^{4}$ ascribed the endoand exothermic peaks for polyhexadecyl methacrylate to the melt and crystallization of hexadecyl side chains. X-ray, IR and other evidence supported this. Then, the endo- and exothermic peaks for other three comb-like polymers should also be ascribed to the melt and crystallization of their hexadecyl side chains.

The four polymers show different DSC traces. The fumarate polymer shows rather broad melting and crystallizing peaks at about $40^{\circ} \mathrm{C}$. The itaconate polymer shows sharp peaks at somewhat lower temperatures than the fumarate polymer. The methacrylate polymer shows peaks at still lower temperatures. The itaconate polymer with one hexadecyl and one methyl ester group shows a sharp melting peak but a weak and broad crystallizing peak.

Figures 2 and 3 show heating and cooling DSC traces for polydialkyl fumarates and itaconates respectively. Alkyl groups are from dodecyl to octadecyl. For both narrowly spaced comb-like polymers, as the number of carbon atoms in the alkyl groups increases from 12 to 18 , the melting and crystallizing peaks appear at higher temperature and have larger areas. This is the same trend with that observed for conventional and widely spaced

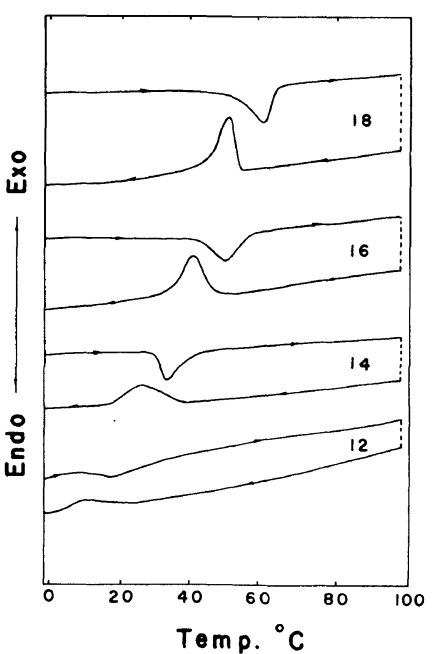

Figure 2. DSC traces for polydialkyl fumarates. Numbers designate the carbon numbers of alkyl side chains.

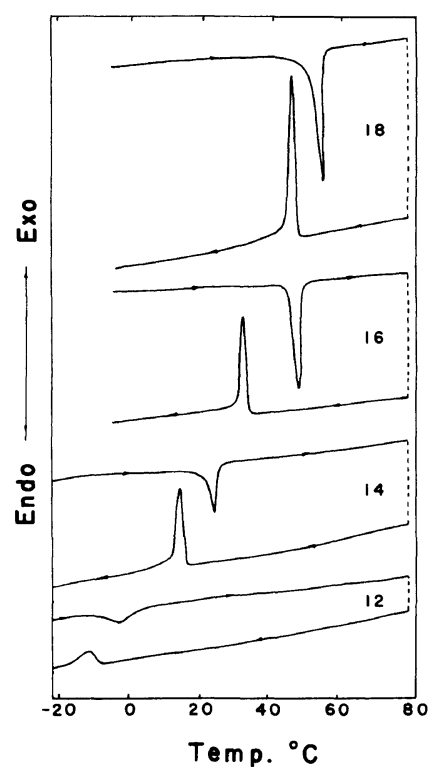

Figure 3. DSC traces for polydialkyl itaconates. Numbers designate the carbon numbers of alkyl side chains.

comb-like polymers. Broad peaks for fumarate polymers and sharp peaks for itaconate polymers are again observed, the reason for this is that fumarate polymers have one ester side chain on every main chain carbon atom and 
Table II. Side-chain crystallization of poly(dialkyl fumarates), poly(dialkyl itaconates), and related polymers

\begin{tabular}{|c|c|c|c|}
\hline \multirow[t]{2}{*}{ Polymer } & $\begin{array}{c}\text { Crystallizing } \\
\text { temperature }^{\mathrm{a}} \\
T_{\mathrm{f}}\end{array}$ & $\begin{array}{c}\text { Heat of } \\
\text { crystallization } \\
\Delta H_{\mathrm{f}}\end{array}$ & $\begin{array}{l}\text { Number of } \\
\text { crystallizing } \\
\mathrm{CH}_{2} \text { groups }\end{array}$ \\
\hline & ${ }^{\circ} \mathrm{C}$ & $\mathrm{kJ}$ repeating unit ${ }^{-1} \mathrm{~mol}^{-1}$ & $N$ \\
\hline \multicolumn{4}{|l|}{ Poly(dialkyl fumarates) } \\
\hline Dodecyl & 10 & 7.5 & 1.2 \\
\hline Tetradecyl & 28 & 18.5 & 3.0 \\
\hline Hexadecyl & 42 & 34.5 & 5.6 \\
\hline Octadecyl & 52 & 43.9 & 7.1 \\
\hline \multicolumn{4}{|l|}{ Poly(dialkyl itaconates) } \\
\hline Dodecyl & -10 & 1.4 & 0.2 \\
\hline Tetradecyl & 13 & 18.5 & 3.0 \\
\hline Hexadecyl & 33 & 31.4 & 5.1 \\
\hline Octadecyl & 46 & 49.8 & 8.1 \\
\hline Poly(hexadecyl methyl itaconate) & 14.5 & 4.7 & 1.5 \\
\hline \multicolumn{4}{|l|}{ Poly(alkyl methacrylates) ${ }^{1}$} \\
\hline Dodecyl & -42 & 1.6 & 0.5 \\
\hline Tetradecyl & -13 & 5.9 & 1.9 \\
\hline Hexadecyl & 11 & 12.4 & 4.0 \\
\hline Octadecyl & 23 & 17.3 & 5.6 \\
\hline
\end{tabular}

a From the cooling DSC peak top.

b Per one alkyl side-chain.

therefore have semi-rigid a structure but itaconate polymers have one methylene group between two quarternary carbon atoms bearing two ester side chains and therefore have a flexible structure. The flexible main chain structure would allow the side chains to crystallize uniformly in length. The rigid structure would be unfavorable for uniform crystallization and give broad peaks in thermal analysis. The influence of rigid and flexible main chain structures on the side chain crystallization was discussed in a previous paper. ${ }^{3}$ It will also be discussed in our next paper which deals with alternating styrene-acrylate comblike copolymers.

Table II shows, as calculated from the cooling peaks in Figure $1-3$, heats of crystallization $\Delta H_{\mathrm{f}}$, crystallizing temperature $T_{\mathrm{f}}$, and the number of crystallizing methylene groups $N$ per one alkyl side chain for four types of comb-like polymers in this study. $N$ values were calculated dividing $\Delta H_{\mathrm{f}}$ by the heat of fusion per $\mathrm{CH}_{2}$ of $n$-alkanes crystallized in the hexagonal packing $\left(3.077 \mathrm{~kJ} \mathrm{CH}_{2}{ }^{-1}\right) .{ }^{9}$ For fumarate and itaconate polymers which have two alkyl side chains in one repeating unit, $N$ values for one side chain were obtained further by dividing by 2 . When compared as a whole, the two narrowly spaced comb-like polymers show a bit larger $N$ values than the conventional ones.

Two narrowly spaced comb-like polymers, polydihexadecyl fumarate and itaconate, showed peaks at $2 \theta=21^{\circ}$ in the X-ray diffraction patterns. They showed sharp single absorption bands at $720 \mathrm{~cm}^{-1}$ in IR spectra. Both results are the same with those observed for the conventional comb-like polymers in the literatures and for the widely spaced comb-like polymers already studied and indicate the hexagonal-packed long alkyl side chains.

Acknowledgment. The authors wish to 
thank Iwata Chemical Industry Co., Ltd. for the research grant and gift of itaconic anhydride.

\section{REFERENCES}

1. K. Yokota, T. Kougo, and T. Hirabayashi, Polym. J., 15, 891 (1983).

2. K. Yokota and T. Hirabayashi, Polym. J., 17, 991 (1985).

3. K. Yokota and T. Hirabayashi, Polym. J., 18, 177 (1986).
4. N. A. Platé and V. P. Shibaev, J. Polym. Sci., Macromol. Rev., 8, 117 (1974).

5. T. Otsu, O. Ito, N. Toyoda, and S. Mori, Makromol. Chem., Rapid Commun., 2, 725 (1981).

6. N. Toyoda and T. Otsu, J. Macromol. Sci.-Chem., A19, 1011 (1983).

7. T. Otsu, T. Yasuhara, K. Shiraishi, and S. Mori, Polym. Bull., 12, 449 (1984).

8. C. S. Marvel and T. H. Shepherd, J. Org. Chem., 24, 599 (1959).

9. S. Nagai and K. Yoshida, Kobunshi Kagaku, 17, 79 (1960). 